review of cranial measurements. The material seems insufficient and fragmentary, and affords imperfect means for judging in a satisfactory way of the exact status and organisation of these people. A final contribution to the linguistics of the subject, by A. S. Gatschet, closes the volume, with a compendious statement of the relations of the tribes of the western coast with a list of forty vocabularies of western languages.

Finally, this handsome volume, in typography, paper, and illustrations, is of irreproachable beauty, and it treats of a field in archæological study of deep interest and wide import.

L. P. GRatacaP

\section{THE SIZE OF ATOMS 1}

\section{II.}

$\mathrm{T}$ making brass, if we mix zinc and copper together we find no very manifest signs of chemical affinity at all ; there is not a great deal of heat developed: the mixture does not become warm, it does not explode. Hence we can infer certainly that contact-electricity action ceases, or does not go on increasing according to the same law, when the metals are subdivided to something like $1 / 100,000,000$ of a centimetre. Now this is an exceedingly important argument. I have more decided data as to the actual magnitude of atoms or molecules to bring before you presently, but I have nothing more decided in giving for certain a limit to supposable smallness. We cannot reduce zinc and copper beyond a certain thickness. without putting them into a condition in which they lose their properties as wholes, and in which, if put together, we should not find the same attraction as we should calculate upon from the thicker plates. I think it is impossible consistently with the knowledge we have of chemical affinities and of the effect of melting zinc and copper together, to admit that a piece of copper or zinc could be divided to a thinness of much less, if at all less, than $\mathrm{r} / 100,000,000$ of a centimetre without separating the atoms or dividing the molecules, or doing away with the composition which constitutes as a whole the solid metal. In short, the structure as it were of bricks, or molecules, or atoms, of which copper and zinc are built up ; cannot be much, if at all, less than $\mathrm{I} / \mathrm{100}, 000,000$ of a centimetre in diameter, and may be considerably greater.

I will now read you a statement from an article which was published thirteen years ago in NATURE. ${ }^{2}$

"Now let a second plate of zinc be brought by a similar " process to the other side of the plate of copper; a second "plate of copper to the remote side of this second plate of "zinc, and so on till a pile is formed consisting of 50,00 I "plates of zinc and 50,000 plates of copper, separated by " 100,000 spaces, each plate and each space $\mathrm{I} / 100,000$ of " a centimetre thick. The whole work done by electric "attraction in the formation of this pile is two centimetre"grammes.

"The whole mass of metal is eight grammes. Hence " the amount of work is a quarter of a centimetre-gramme "per gramme of metal. Now 4030 centimetre-grammes of " work, according to Joule's dynamical equivalent of heat, "is the amount required to warm a gramme of zinc or " copper by one degree Centigrade. Hence the work done "by the electric attraction could warm the substance by " only I/16,120 of a degree. But now let the thickness of "each piece of metal and of each intervening space be " $\mathrm{I} / 100,000,000$ of a centimetre instead of $\mathrm{I} / \mathrm{100}, 000$. "The work would be increased a millionfold unless " $\mathrm{I} / \mathrm{I} 00,000,000$ of a centimetre approaches the small" ness of a molecule. The heat equivalent would therefore "be enough to raise the temperature of the material by

${ }^{x}$ A lecture delivered by Sir William Thomson at the Royal Institution, on Friday, February 2. Revised by the Author. Continued from p. 205.

${ }^{2}$ See article "On the Size of Atoms," published in NATURE, vol.i. T. 55I; printed in Thomson and Tait's "Natural Philosophy," second edition, r883, vol. i. part 2, Appendix $\mathrm{F}$.
" $62^{\circ}$. This is barely, if at all, inadmissible, according to " our present knowledge, or, rather, want of knowledge, " regarding the heat of combination of zinc and copper. "But suppose the metal plates and intervening spaces to " be made yet four times thinner, that is to say, the thick"ness of each to be $1 / 400,000,000$ of a centimetre. The "work and its heat equivalent will be increased sixteen"fold. It would therefore be 990 times as much as that "required to warm the mass by $\mathrm{I}^{\circ} \mathrm{C}$., which is very much "more than can possibly be produced by zinc and copper "in entering into molecular combination. Were there in "reality anything like so much heat of combination as this, " a mixture of zinc and copper powders would, if melted in "any one spot, run together, generating more than heat " enough to melt each throughout; just as a large quantity " of gunpowder if ignited in any one spot burns throughout "without fresh application of heat. Hence plates of zinc "and copper of $1 / 300,000,000$ of a centimetre thick, " placed close together alternately, form a near approxima"tion to a chemical combination, if indeed such thin plates "could be made without splitting atoms."

Similar conclusions result from that curious and most interesting phenomenon, the soap-bubble. Philosophers old and young who occupy themselves with soap-bubbles, have one of the most interesting subjects of physical science to admire. Blow a soap-bubble and look at it, -you may study all your life perhaps and still learn lessons in physical science from it. You will now see on the screen the image of a soap-film in a ring of metal. The light is reflected from the film filling that ring, and focused on the screen. It will show, as you see, colours analogous to those of Newton's rings. As you see the image it is upside down. The liquid streams down (up in the image) and thins away from the highest point of the film. First we see that brilliant green colour. It will become thinner and thinner there, and will pass through beautiful gradations of colour till you see, as now, a deep red, then much lighter, till it becomes a dusky, yellowish white, then green, and blue, and deep violet, and lastly black, but after you see the black spot it very soon bursts. The film itself seems to begin to lose its tension, when it gets considerably less than a quarter of the wave-length of yellow light, which is the thickness for the dusky white, preceding the final black. When you are washing your hands, you may make and deliberately observe a film like this, in a ring formed by the forefingers and thumbs of two hands, and watch the colours. Whenever you begin to see a black spot or several black spots, the film soon after breaks. The film retains its strength until we come to the black spot, where the thickness is clearly much less than $\mathrm{I} / 60,000$ of a centimetre, which is the thickness of the dusky white.

Newton, in the following passage in his "Optics" (pp. I87 and 191 of edition 172I, Second Book, Part I.), tells more of this important phenomenon of the black spot, than is known to many of the best of modern observers.

"Obs. 17.-If a bubble be blown with water, first made " tenacious by dissolving a little soap in it, it is a common " observation that after a while it will appear tinged with a "variety of colours. To defend these bubbles from being " agitated by the external air (whereby their colours are " irregularly moved one among another so that no accurate " observation can be made of them), as soon as I had " blown any of them I covered it with a clear glass, and by " that means its colours emerged in a very regular order, "like so many concentric rings encompassing the top of " the bubble. And as the bubble grew thinner by the con" tinual subsiding of the water, these rings dilated slowly " and overspread the whole bubble, descending in order to " the bottom of it, where they vanished successively. In "the meanwhile, after all the colours were emerged at the " top, there grew in the centre of the rings a small round "black spot like that in the first observation, which con" tinually dilated itself, till it became sometimes more than 
" one-half or three-quarters of an inch in breadth before the "bubble broke. At first I thought there had been no light "reflected from the water in that place, but observing it "more curiously I saw within it several smaller round "spots, which appeared much blacker and darker than the "rest, whereby I knew that there was some reflection at the " other places which were not so dark as those spots. And "by farther trial I found that I could see the images of "some things (as of a candle or the sun) very faintly re"flected, not only from the great black spot, but also from " the little darker spots which were within it.

"Obs. 18.- If the water was not very tenacious, the "black spots would break forth in the white without any "sensible intervention of the blue. And sometimes they "would break forth within the precedent yellow, or red, "or perhaps within the blue of the second order, before " the intermediate colours had time to display themselves."

Now I have a reason, an irrefragable reason, for saying that the film cannot keep up its tensile strength to $\mathrm{I} / \mathrm{I00}, 000,000$ of a centimetre, and that is, that the work which would be required to stretch the film a little more than that, would be enough to drive it into vapour.

The theory of capillary attraction shows, that when a bubble-a soap-bubble for instance-is blown larger and larger, work is done by the stretching of a film which resists extension, as if it were an elastic membrane with a constant contractile force. This contractile force is to be reckoned as a certain number of units of force per unit of breadth. Observation of the ascent of water in capillary tubes, shows that the contractile force of a thin film of water, is about sixteen milligrammes weight per millimetre of breadth. Hence the work done in stretching a water film to any degree of thinness, reckoned in millimetre-milligrammes, is equal to sixteen times the number of square millimetres by which the area is augmented, provided the film is not made so thin that there is any sensible diminution of its contractile force. In an article "On the Thermal Effect of Drawing out a Film of Liquid," published in the Proceedings of the Royal Society for April, I858, I have proved from the second law of thermodynamics that about half as much more energy, in the shape of heat, must be given to the film, to prevent it from sinking in temperature while it is being drawn out. Hence the intrinsic energy of a mass of water in the shape of a film kept at constant temperature, increases by twenty-four milligramme-millimetres for every square millimetre added to its area.

Suppose then a film to be given with a thickness of a millimetre, and suppose its area to be augmented ten thousand and one fold: the work done per square millimetre of the original film, that is to say, per milligramme of the mass, would be 240,000 millimetre-milligrammes. The heat equivalent of this is more than half a degree Centigrade $\left(0.57^{\circ}\right)$ of elevation of temperature of the substance. The thickness to which the film is reduced on this supposition, is very approximately $\mathrm{r} / \mathrm{I} 0$, , o o o millimetre. The commonest observation on the soap-bubble, shows that there is no sensible diminution of contractile force, by reduction of the thickness to $1 / 10,000$ of a millimetre; inasmuch as the thickness which gives the first maximum brightness, round the black spot seen where the bubble is thinnest, is only about $1 / 8,000$ of a millimetre.

The very moderate amount of work shown in the preceding estimates, is quite consistent with this deduction. But suppose now the film to be farther stretched, until its thickness is reduced to $1 / 10,000,000$ of a millimetre i $1 / 100,000,000$ of a centimetre). The work spent in doing :his is two thousand times more than that which we have iust calculated. The heat equivalent is 280 times the zuantity required to raise the temperature of the liquid by one degree Centigrade. This is far more than we can zdmit as a possible amount of work done in the extension of a liquid film. It is more than half the amount of work, which if spent on the liquid, would convert it into vapour at ordinary atmospheric pressure. The conclusion is unavoidable, that a water-film falls off greatly in its contractile force, before it is reduced to a thickness of I/10, 000,000 of a millimetre. It is scarcely possible, upon any conceivable molecular theory, that there can be any considerable falling off in the contractile force, as long as there are several molecules in the thickness. It is therefore probable that there are not several molecules in a thickness of $1 / 10,000,000$ of a millimetre of water.

Now when we are considering the subdivision of matter, look at those beautiful colours which you see in this little casket, left, I believe, by Prof. Brand to the Royal Institution. It contains polished steel bars, coloured by having been raised to different degrees of heat, as in the process of annealing hard-tempered steel. These colours, produced by heat on other polished metals besides steel, are due to thin films of transparent oxide, and their tints, as those of the soap-bubble and of the thin space of air in "Newton's rings," depend on the thickness of the film, which, in the case of oxidisable metals, forms by combination with the oxygen of the air, under the influence of heat - a true surface-burning.

You are all familiar with the brilliant and beautifully distributed fringes of heat-colours on polished steel grates and fire-irons, escaping that unhappy rule of domestic æsthetics, which too often keeps those articles glittering and cold and useless, instead of letting them show the exquisite play of warm colouring, naturally and inevitably brought out, when they are used in the work which is their reason for existence. The thickness of the film of oxide which gives the first perceptible colour, a very pale orange or buff tint, due to the enfeeblement or extinction of violet light and enfeeblement of blue, and less enfeeblement of the other colours in order, by interference of the reflections from the two surfaces of the film, is about I/100,000 of a centimetre, being something less than a quarter wave-length of violet light in the oxide.

The exceedingly searching and detective efficacy of electricity comes to our aid here, and by the force as it were spread through such a film, proves to us the existence of the film when it is considerably thinner than that $\mathrm{I} / \mathrm{IO0}, 000$ of a centimetre, when in fact it is so very thin as to produce absolutely no perceptible effect on the reflected light, that is to say, so thin as to be absolutely invisible. If in the apparatus for measuring contact electricity, of which the drawing is before you (NATURE, vol. xxiii. p. 567), two plates of freshly polished copper be placed in the Volta condenser, a very perfect zero of effect is obtained. If, then, one of the plates be taken out, heated slightly by laying it on a piece of hot iron, and then allowed to cool again and replaced in the Volta condenser, it is found that negative electricity becomes condensed on the surface thus treated, and positive electricity on the bright copper surface facing it, when the two are in metallic connection. If the same process be repeated with somewhat higher temperatures, or somewhat longer times of exposure to it, the electrical difference is augmented. These effects are very sensible before any perceptible tint appears on the copper surface as modified by heat. The effect goes on increasing with higher and higher temperatures of the heating influence, until oxide-tints begin to appear, commencing with buff, and going on through a ruddier colour to a dark-blue slate colour, when no farther heating seems to augment the effect. The greatest contact-electricity effect which I thus obtained between a bright freshly polished copper surface and an opposing face of copper, rendered almost black by oxidation, was such as to require for the neutralising potential in my mode of experimenting ${ }^{1}$ about onehalf of the potential of a Daniell's cell.

I First described in a letter to Joule, published in the Proceedings of the Literary and Philosophical Society of Manchester of Jan, 21, 1862, where also I first pointed out the demonstration of a limit to the size of molecules fully described in the article of NATURE (vol. xxiii. p. 567), referred to above. 
Some not hitherto published experiments with polished silver plates, which I made fifteen years ago, showed me very startlingly, an electric influence from a quite infinitesimal whiff of iodine vapour. The effect on the contact-electricity quality of the surface, seems to go on continuously from the first lodgment, to all other tests quite imperceptible, of a few atoms or molecules of the attacking substance (oxygen, or iodine, or sulphur, or chlorine, for example), and to go on increasing until some such thickness as $1 / 30,000$ or $1 / 40,000$ of a centimetre is reached by the film of oxide or iodide, or whatever it may be that is formed.

The subject is one that deserves much more of careful experimental work and measurement than has hitherto been devoted to it. I allude to it at present to point out to you how it is that by this electric action, we are enabled as it were to sound the depth, of the ocean of molecules attracted to the metallic surface, by the vapour or gas entering into combination with it.

When we come to thicknesses of considerably less than a wave-length, we find solid metals becoming transparent. Through the kindness of Prof. Dewar, I am able to show you some exceedingly thin films, of measured thicknesses of platinum, gold, and silver, placed on glass plates. The platinum is of $I^{\circ} 9 \times$ Io thickness, and is quite opaque ;

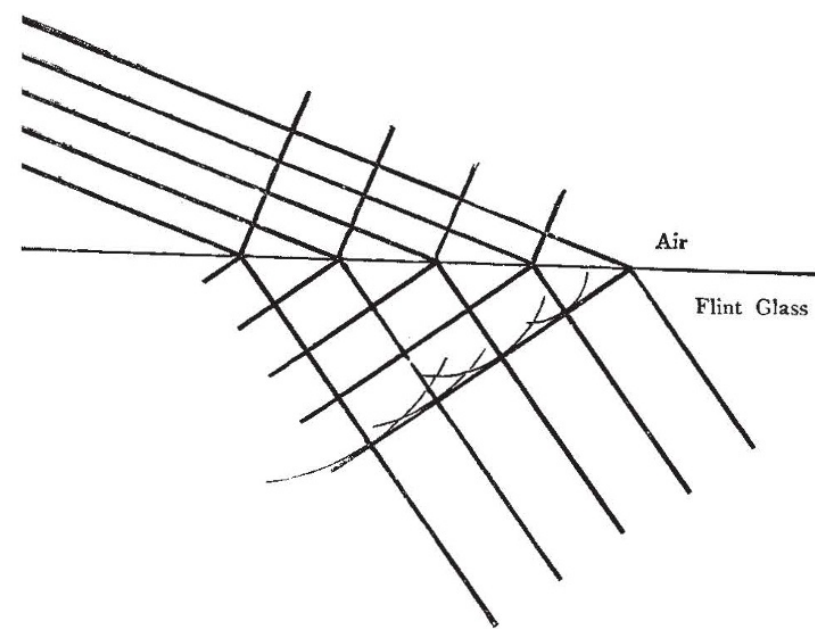

FIG. 3.-Diagram of Huyghen's construction fur wave front of refracted light. Drawn for light passing from air to flint glass.

but here is a gold film of about the same thickness, which is transparent to the electric light, as you see, and transmits the beautiful green colour, which you see on the screen. The thickness of this gold ( 19 , or nearly 2 ) is just half the wave-length of violet light in air. This transparent gold, transmitting green light to the screen as you see, at the same time reflects yellow light to the ceiling. Now I will show you the silver. It is thinner, being only $1.5 \times 10^{-5}$ of a centimetre thick, or $3 / 8$ of the air-wavelength of violet light. It is quite opaque to the electric light, so far as our eyes allow us to judge, and reflects all the light up to the ceiling. It is not wonderful that it should be opaque; we might wonder if it were otherwise; but there is an invisible ultra-violet light of a small range of wave-iengths, including a zinc-line of air wavelength $3.4 \times 10^{-5}$, which this silver film transmits. For that particular light the silver film of $1.5 \times 10^{-5}$ thickness is transparent. The image which you now see on the screen, is a magic lantern representation of the selfphotographed spectrum, of light that actually came through that silver. You see the zinc-line very clear across it near its middle. Here then we have gold and silver transparent. The silver is opaque for all except that very definite light of wave-lengths from about 3.07 to 3.32

The different refrangibility of different colours, is a result of observation, of vital importance in the question of the size of atoms. You now see on the screen before you a prismatic spectrum; a well-known phenomenon produced by the differences of the refractions of the dif ferent colours, in traversing the prism. The explanation of it in the undulatory theory of light, has taxed the powers of mathematicians to the utmost. Look first, however, to what is easy, and made clear by that diagram (Fig. 3) before you ; and you will easily understand that refraction depends on difference of velocity of propagation of light, in the two transparent mediums concerned. The

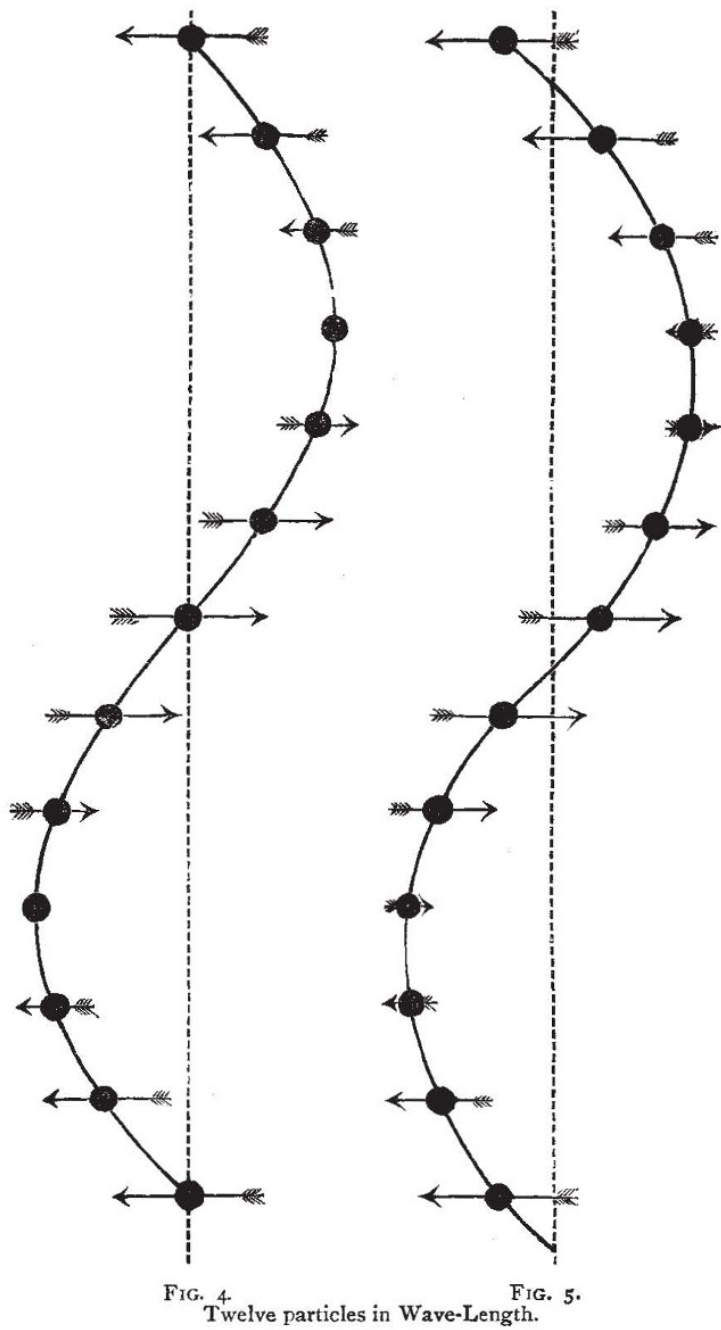

angles in the diagram are approximately correct, for refraction at an interface between air or vacuum and flint glass ; and you see that in this case, the velocity of propagation is less in the denser medium. The more refractive medium (not always the denser) of the two, has the less velocity for light transmitted through it. The "refractive index" of any transparent medium, is the ratio of the velocity of propagation in the ether, to the velocity of propagation in the transparent substance.

Now, that the velocity of the propagation of light should be different in different mediums, and should in most cases be smaller in the denser than in the less dense medium, is quite what we should, according to dynamical principles, expect from any conceivable constitution of the 
luminiferous ether and of palpable transparent substance. But that the velocity of propagation in any one transparent substance, should be different for light of different colours, that is to say, of different periods of vibration, is not what we should expect; and could not possibly be the fact if the medium is homogeneous, without any limit as to the smallness of the parts of which the qualities are compared. The fact that the velocity of propagation does depend on the period, gives what I believe to be irrefragable proof, that the substance of palpable transparent matter, such as water, or glass, or the bisulphuret of carbon of this prism whose spectrum is before you, is not infinitely homogeneous; but that on the contrary, if contiguous portions of any such medium, any medium in fact which can give the prismatic colours, be examined at intervals not incomparably small in comparison with the wave-lengths, utterly heterogeneous quality will be discovered; such heterogeneousness as that which we understand in palpable matter, as the difference between solid and fluid; or between substances differing enormously in density; or such heterogeneousness as differences of velocity and direction of motion, in different positions of a vortex ring in an homogeneous liquid; or such differences of material occupying the space examined, as we find in a great mass of brick building when we pass from brick to brick through mortar (or through void, as we too often find in Scotch-built domestic brick chimneys).

Cauchy was I believe the first of mathematicians or naturalists, to allow himself to be driven to the conclusion, that the refractive dispersion of light can only be accounted for, by a finite degree of molecular coarsegrainedness, in the structure of the transparent refracting matter ; and as, however we view the question, and however much we may feel compelled to differ, from the details of molecular structure and molecular inter-action assumed by Cauchy, we remain more and more surely fortified in his conclusion, that finite grainedness of transparent palpable matter, is the cause of the difference of the velocity of different colours of light propagated through it, we must regard Cauchy as the discoverer of the dynamical theory of the prismatic colours.

But now we come to the grand difficulty of Cauchy's theory ${ }^{1}$ look at this little table ('Table II.), and you will see

TABLE II.-Velocity $(V)$ according to Number $(N)$ of Particles
in Wave Length

\begin{tabular}{|c|c|}
\hline$N$ & $V\left(=\operatorname{xo0} \frac{\sin (\pi / N)}{\pi / N}\right)$ \\
\hline 2 & 63.64 \\
4 & 90.03 \\
8 & 97.45 \\
12 & 98.86 \\
16 & 99.36 \\
20 & 99.59 \\
$\infty$ & 100.00
\end{tabular}

in the heading, the formula which gives the velocity, in terms of the number of particles to the wave-length, supposing the medium to consist of equal particles arranged in cubic order, and each particle to attract its six nearest neighbours, with a force varying directly as the excess of the distance between them, above a certain constant line (the length of which is to be chosen, according to the degree of compressibility possessed by the elastic solid, which we desire to represent by a crowd of mutually interacting molecules). If you suppose particles of real matter arranged in the cubic order, and six steel wire spiral springs or elastic indiarubber bands, to

"For an account of the dynamical theory of the "Dispersion of Light," see "View of the Undulatory Theory as Applied to the Dispersion of Light," by the Rev. Baden Powell, M.A., \&c. (London: $x 8_{4} \mathrm{I}$ ) be hooked on to each particle and stretched between it and its six nearest neighbours, the postulated force may be produced in a model with all needful accuracy; and if we could but successfully wish the theatre of the Royal Institution, conveyed to the centre of the earth, and kept there for five minutes, I should have great pleasure in showing you a model of an elastic solid thus constituted, and showing you waves propagated through it, as are waves of light in the luminiferous ether. Gravity is the inconvenient accident of our actual position, which prevents my showing it to you here just now. But instead, you have these two wave-models (see Fig. 2 above), each of which shows you the displacements and motions of a

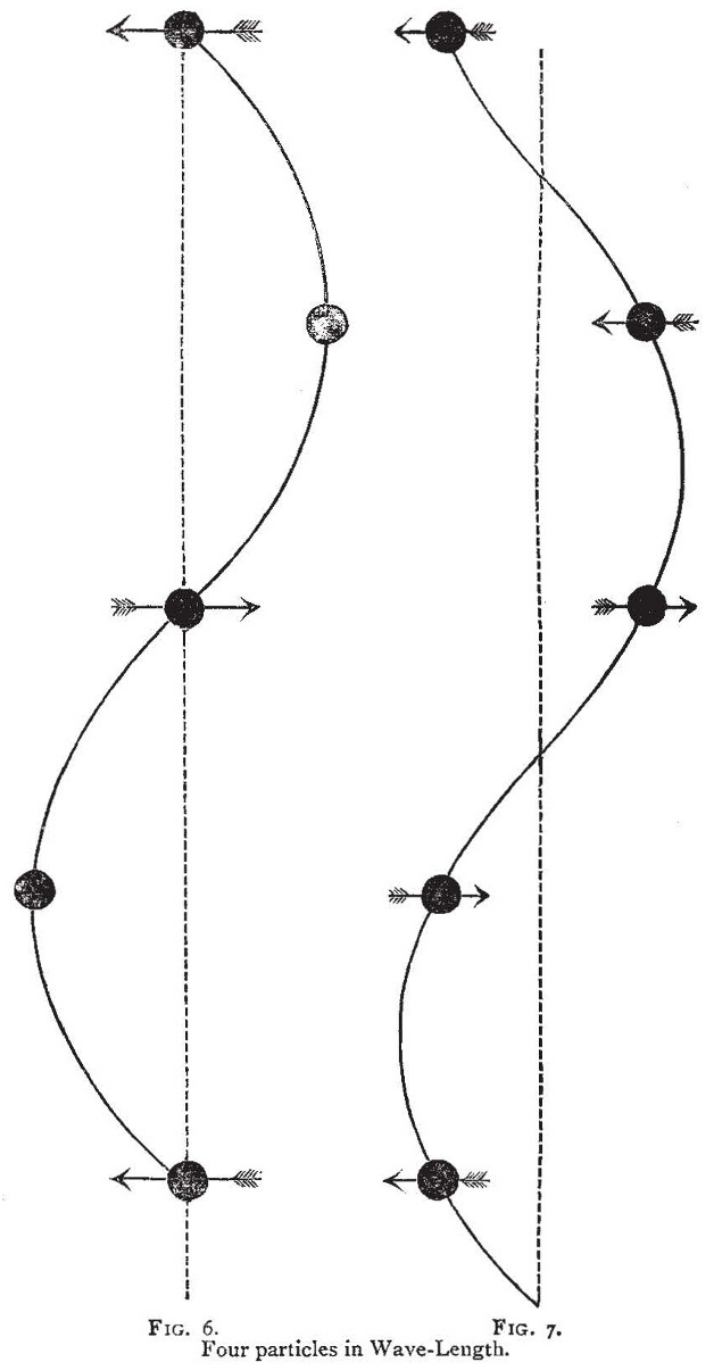

line of particles, in the propagation of a wave through our imaginary three-dimensional solid; the line of molecules chosen being those which, in equilibrium, are in one direct straight line of the cubic arrangement, and the supposed wave having its wave front perpendicular to this line, and the direction of its vibration, the direction of one of the other two direct lines of the cubic arrangement.

You have also before you this series of diagrams (Figs. 4 to 9) of waves in a molecularly-constituted elastic solid. These two diagrams (Figs. 4 and 5) illustrate a wave in which there are twelve molecules in the wavelength; this one (Fig. 4) showing (by the length and position of the arrows) the magnitude and direction of velocity of each molecule, at the instant when one of the 
molecules is on the crest of the wave, or has reached its maximum displacement; that one (Fig. 5) showing the magnitude and direction of the velocities, after the wave has advanced such a distance, as (in this case equal to onetwenty-fourth of the wave-length) to bring the crest of the wave to midway between two molecules. This pair of diagrams (Figs. 6 and 7) shows the same for waves having four molecules in the wave-length, and this pair (Figs. 8 and 9) for a wave having two molecules in the wavelength.

The more nearly this critical case is approached, that is to say the shorter the wave-length, down to the limit of twice the distance from molecule to molecule, the less ecomes the difference between the two configurations of

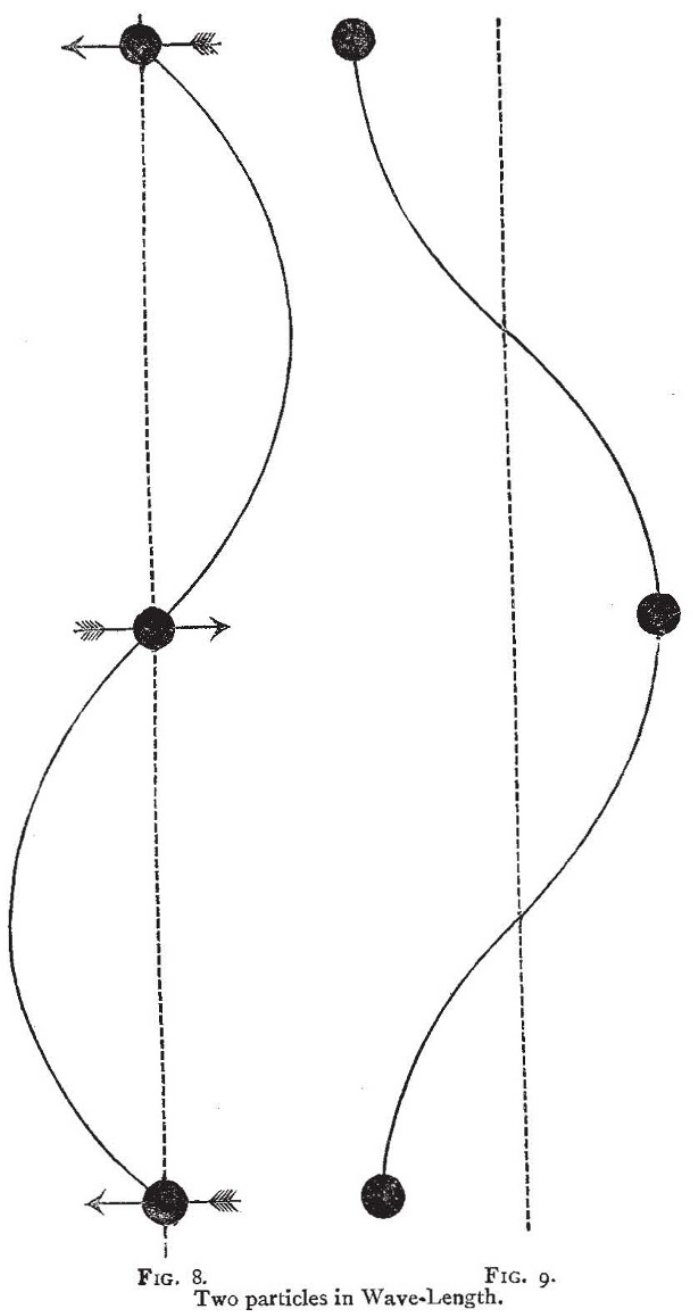

motion, constituted by waves travelling in opposite directions. In the extreme or critical case, the difference is annulled, and the motion is not a wave-motion, but a case of what is often called "standing vibration." Before I conclude this evening, I hope to explain in detail the kind of motion which we find instead of wave-motion (become mathematically imaginary), when the vibrational period of the exciter is anything less than the critical value; because this case is of extreme importance and interest in physical optics, according to Stokes' hitherto unpublished explanation of phosphorescence.

This supposition of each molecule acting with direct force only on its nearest neighbour, is not exactly the postulate on which Cauchy works. He supposes each molecule to act on all around it, according to some law of rapid decrease as the distance increases; but this must make the influence of coarse-grainedness on the velocity of propagation smaller than it is on the simple assumption, realised in the models and diagrams before you, which therefore represents the extreme limit of the efficacy of Cauchy's unmodified theory to explain dispersion.

Now, by looking at the little table (Table II.) of calculated results, you will see that with as few as 20 molecules in the wave-length, the velocity of propagation is $99 \frac{1}{2}$ per cent. of what it would be with an infinite number of molecules; hence the extreme difference of propagational velocity, accountable for by Cauchy's unmodified theory in its idealised extreme of mutual action limited to nearest neighbours, amounts to $\mathrm{I} / 200$. Now look at this table (Table III.) of refractive indices, and you see that the difference of velocity of red light (A), and of violet light $(\mathrm{H})$, amounts in carbon disulphide to $\mathrm{I} / \mathrm{I} 7$; in dense flint glass to nearly $1 / 30$; in hard crown glass to $1 / 73$; and in water and alcohol to rather more than $\mathrm{I} / \mathrm{Ioo}$. Hence, none of these substances can have so many as 20 molecules in the wave-length, if dispersion is to be accounted for by Cauchy's unmodified theory, and by looking back to the little table of calculated results (Table II.), you will

TABLE III. - Table of Refractive Indices.

\begin{tabular}{|c|c|c|c|c|c|}
\hline \multirow{2}{*}{$\begin{array}{l}\text { Line of } \\
\text { Spectrum. }\end{array}$} & \multicolumn{5}{|c|}{ Material. } \\
\hline & $\begin{array}{c}\text { Hard Crown } \\
\text { Glass. }\end{array}$ & $\begin{array}{l}\text { Extra dense } \\
\text { Flint Glass. }\end{array}$ & $\begin{array}{l}\text { Water at } \\
15^{\circ} \mathrm{C} \text {. }\end{array}$ & $\begin{array}{l}\text { Carbon } \\
\text { Disulphide } \\
\text { at } 1 \mathrm{I}^{\circ} \mathrm{C} .\end{array}$ & $\begin{array}{l}\text { Alcohol } \\
\text { at } 15^{\circ} \mathrm{C} \text {. }\end{array}$ \\
\hline A & $1 \cdot 5118$ & $x \cdot 639 x$ & I. 3284 & $\mathrm{I} \cdot 6 \mathrm{I} 42$ & $\mathrm{I} \cdot 3600$ \\
\hline B & I. 5136 & 1.6429 & I. 3300 & 1.6207 & $I \cdot 3612$ \\
\hline C & I. 5146 & I.6449 & I. 3307 & 1.6240 & I. $362 \mathrm{r}$ \\
\hline D & I. 5171 & I. 6504 & I. 3324 & I. 6333 & $\mathrm{I} \cdot 3638$ \\
\hline $\mathrm{E}$ & $1 \cdot 5203$ & I. 6576 & I. 3347 & $1 \cdot 6465$ & $x \cdot 366 x$ \\
\hline $\mathrm{b}$ & 1.5210 & I. 6591 & - & - & \\
\hline$F$ & $x \cdot 523 I$ & I 6442 & $I \cdot 3366$ & I. 6584 & I' 3683 \\
\hline G & 1.5283 & 1.6770 & $1 \cdot 3402$ & I. 6836 & $\mathrm{I} \cdot 3720$ \\
\hline $\mathrm{h}$ & 1.5310 & 1.6836 & - & - & \\
\hline $\mathrm{H}$ & I. 5328 & I. 6886 & I'343I & $I \cdot 7090$ & $\mathrm{I}^{*} 375^{\mathrm{I}}$ \\
\hline
\end{tabular}

The numbers in the first two columns were determined by Dr. Hopkinson those in the last three by Messrs. Gladstone and Dale. The index of refraction of air for light near the line $\mathrm{E}$ is $\mathrm{x} \cdot 000294$.

see that there could not be more than twelve molecules in the wave-length of violet light in water or alcohol ; sty ten in hard crown glass; eight in flint glass; and in carbon disulphide actually not more than four molecules in the wave-length, if we are to depen' upon Cauchy's unmodified theory for the explanation of dispersion. So large coarse-grainedness of ordinary transparent bodies, solid or fluid, is quite untenable. Before I conclude 1 intend to show you, from the kinetic theory of gases, a superior iimit to the size of molecules, according to which, in glass or in water, there is probably something like 600 molecules to the wave-length; and almost certainly not fewer than two, or three, or four hundred. But even without any such definite estimate of a superior limit to the size of molesules, there are many reasons against the admission that it is probable or possible, there can be only four, or five, or six to the wave-length. The very drawing by Nobert of 4,000 lines on a breadth of a millimetre, or at the rate of 40,000 to the centimetre, or about two to the ether wave-length of blue (F) light, ${ }^{1}$ seems quite to negative the idea of any such possibility, of only five or six molecules to the wave-length, even if we were not to declare against it from theory and observation of the reflection of light from polished surfaces.

\section{(To be continued.)}

I Loschmidt, "quoting from the Zollvereins department of the London International Exhibition of $186_{2}$, page 83, and from Harting "On the Microscope,' page 88r,"' Sitzungsberichte der Wiener Akademie Math. Phys. x865. vil. lii. 